\title{
The irregularity of two types of trees
}

\author{
Jianxi Li $\|^{*} \quad$ Yang Liu ${ }^{1} \quad$ Wai Chee Shiu ${ }^{2}$ \\ 1 School of Mathematics and Statistics, Minnan Normal University, Zhangzhou, Fujian, P.R. China \\ 2 Department of Mathematics, Hong Kong Baptist University, Kowloon Tong, Hong Kong, P.R. China.
}

received $23^{\text {rd }}$ June 2015, revised $27^{\text {th }}$ Mar. 2016, accepted $2^{\text {nd }}$ June 2016.

The irregularity of a graph $G$ is defined as the sum of weights $|d(u)-d(v)|$ of all edges $u v$ of $G$, where $d(u)$ and $d(v)$ are the degrees of the vertices $u$ and $v$ in $G$, respectively. In this paper, some structural properties on trees with maximum (or minimum) irregularity among trees with given degree sequence and trees with given branching number are explored, respectively. Moreover, the corresponding trees with maximum (or minimum) irregularity are also found, respectively.

Keywords: Irregularity, trees, degree sequence, branching number.

\section{Introduction}

Let $G$ be a simple connected graph with vertex set $V(G)$ and edge set $E(G)$. Its order is $|V(G)|$, denoted by $n$, and its size is $|E(G)|$, denoted by $m$. For $v \in V(G)$, let $N_{G}(v)$ (or $N(v)$ for short) be the set of neighbors of $v$ in $G$, and $d_{G}(v)=\left|N_{G}(v)\right|$ (or $d(v)$ for short) be the degree of $v$ in $G$. A pendent vertex of $G$ is a vertex of degree 1 . Let $\Delta_{1}(G), \Delta_{2}(G)$ and $\delta(G)$ (or $\Delta_{1}, \Delta_{2}$ and $\delta$ for short) be the largest, second largest and minimum degrees of $G$, respectively.

A graph whose all vertices have equal degrees is said to be regular, otherwise it is irregular. Albertson [3] defined the imbalance of an edge $u v \in E(G)$ as $|d(u)-d(v)|$ and the irregularity of $G$ as

$$
i r r=i r r(G)=\sum_{u v \in E(G)}|d(u)-d(v)|
$$

where the summation is over all (unordered) edges $u v$ in $G$.

The problem of determining the graph with maximum (or minimum) irregularity (or estimating bounds on $\operatorname{irr}(G)$ ) among some classes of graphs is of great interest. Hansen and Mélot [6] determined the maximum value for the irregularity of graphs of order $n$ with $m$ edges and constructed the corresponding graph which attaining this value; Henning and Rautenbach [7] explored the structural properties on bipartite graphs with maximum irregularity. Various upper bounds on the irregularity of some classes of graphs, such as $K_{r+1}$-free graphs, bipartite graphs, triangle-free graphs were deduced in [14, 3, 1],

\footnotetext{
* This work is partially supported by NSF of China (Nos.11101358, 61379021, 11471077); NSF of Fujian (Nos.2014J01020, 2015J01018, 2016J01673); China Postdoctoral Science Foundation (No.2014M551831); General Research Fund of Hong Kong and Faculty Research Grant of Hong Kong Baptist University.
}

1365-8050 @ 2016 Discrete Mathematics and Theoretical Computer Science (DMTCS), Nancy, France 
respectively. In particular, Zhou [14] established the relationship between $\operatorname{irr}(G)$ and $Z_{1}(G)$, and respectively determined the graphs with maximum irregularity among trees and unicyclic graphs with a given number of pendent vertices, where $Z_{1}(G)=\sum_{v \in V(G)} d_{G}^{2}(v)$ is the first Zagreb index of $G$. Recently, Luo and Zhou [9] determined the graphs with maximum irregularity among trees and unicyclic graphs with given matching number, respectively. More results on imbalance, the irregularity of a graph can be found in [5, 10, 2, 11].

A positive integer sequence $\pi=\left\{d_{1}, d_{2}, \cdots, d_{n}\right\}$ is called the degree sequence of $G$ if $d_{i}=d\left(v_{i}\right)$ for $v_{i} \in V(G), i=1, \cdots, n$. Throughout this paper, we order the vertex degrees in non-increasing order, i.e., $d_{1} \geq d_{2} \geq \cdots \geq d_{n}$. Also, a sequence $\pi=\left\{d_{1}, d_{2}, \cdots, d_{n}\right\}$ is called a tree degree sequence if there exists a tree $T$ having $\pi$ as its degree sequence. Furthermore, the sequence $\pi=\left\{d_{1}, d_{2}, \cdots, d_{n}\right\}$ is a degree sequence of a tree of order $n$ if and only if $\sum_{i=1}^{n} d_{i}=2(n-1)$ [4]. For a tree $T$, a vertex of degree 1 is also called a leaf (or a pendent vertex); a vertex $v$ with $d(v) \geq 3$ is called a branching vertex. The branching number of $T$, denoted by $k(T)$, is the number of those vertices $v \in V(T)$ with $d(v) \geq 3$. For convenience, the degree sequence of $T(\pi(T))$ is the sequence of the degrees (in descending order) of non-leaf vertices. Let $\mathscr{T}_{n}^{\pi}$ and $\mathscr{B}_{n}^{k}$ be the sets of trees of order $n$ with degree sequence $\pi$ and $k$ branching vertices, respectively. Let $T_{\max }^{\pi}$ (or $T_{\min }^{\pi}$ ) be the tree which has maximum (or minimum) irregularity among trees in $\mathscr{T}_{n}^{\pi}$, and $B_{\max }^{k}$ (or $B_{\min }^{k}$ ) be the tree which has maximum (or minimum) irregularity among all trees in $\mathscr{B}_{n}^{k}$.

In the present paper, we explore some properties on $T_{\max }^{\pi}$ (or $T_{\min }^{\pi}$ ), and find the corresponding trees $T_{\max }^{\pi}\left(\right.$ or $\left.T_{\min }^{\pi}\right)$, as well as $B_{\max }^{k}\left(\right.$ or $\left.B_{\min }^{k}\right)$.

\section{Preliminaries}

We use $G-u v$ to denote the graph obtained by deleting the edge $u v \in E(G)$ from $G$. Similarly, $G+u v$ is the graph obtained by adding an edge $u v \notin E(G)$ to $G$.
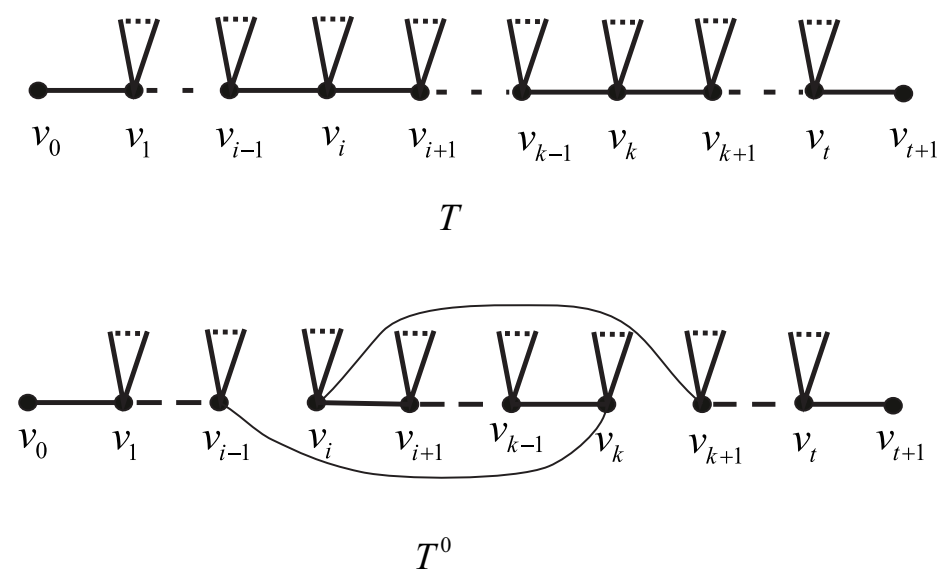

Fig. 1: A new path in $T^{0}$ obtained from $T$ by $S\left(v_{i}, v_{k}\right)$ on the path $v_{0} v_{1} v_{2} \cdots v_{t} v_{t+1}$.

Consider a path $v_{0} v_{1} v_{2} \cdots v_{t} v_{t+1}$ in a tree $T$, where $d_{T}\left(v_{0}\right)=d_{T}\left(v_{t+1}\right)=1$, let $T^{0}$ be a new tree 
obtained from $T$ by reversing the order of the components attached to $v_{i}, v_{i+1}, \cdots, v_{k}$. That is $T^{0}=$ $T-v_{i-1} v_{i}-v_{k} v_{k+1}+v_{i-1} v_{k}+v_{i} v_{k+1}$. Clearly, $T^{0}$ and $T$ have the same degree sequence. This operation is denoted by $S\left(v_{i}, v_{k}\right)$ on the path $v_{0} v_{1} v_{2} \cdots v_{t} v_{t+1}$. The process of this operation is shown in Fig. 1

Let $e=u v$ be an edge of a graph $G$. Let $G^{\prime}$ be the graph obtained from $G$ by contracting the edge $e$ into a new vertex $u^{\prime}$ and adding a new pendent edge $u^{\prime} v^{\prime}$, where $v^{\prime}$ is a new pendent vertex. We say that $G^{\prime}$ is obtained from $G$ by separating an edge $u v$ (shown in Fig. 2).

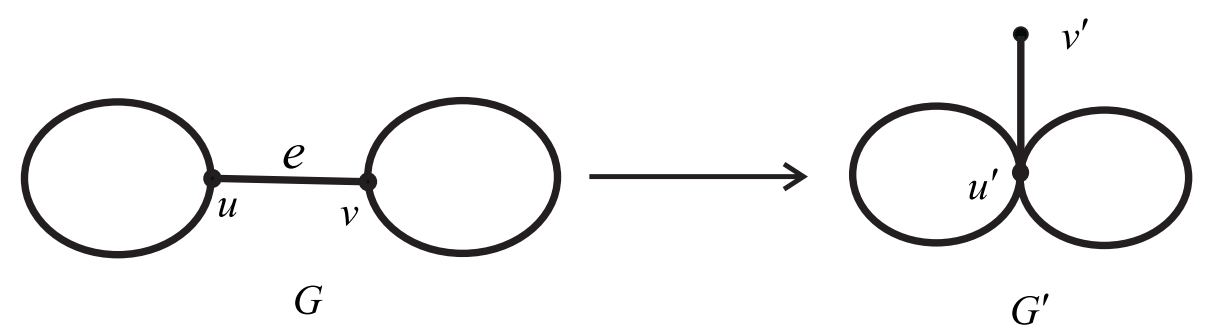

Fig. 2: $G^{\prime}$ is obtained from $G$ by separating an edge $u v$.

Lemma 2.1 ([|8]) For $e=u v \in E(G)$, let $G^{\prime}$ be the graph obtained from $G$ by separating an edge uv. If $d_{G}(u) \geq d_{G}(v)$ for any $v \in N_{G}(u)$, then we have $\operatorname{irr}\left(G^{\prime}\right)>\operatorname{irr}(G)$.

Lemma 2.2 For positive integer $x \leq \frac{n-2}{2}$, the function $f(n, x)=n^{2}+(1-4 x) n+4 x^{2}-2$ is monotonically decreasing on $x$.

Proof: Consider the derivative on $x$ of the function $f(n, x)$. Then we have

$$
\frac{d(f(n, x))}{d x}=8 x-4 n \leq 8 \times \frac{n-2}{2}-4 n=-8<0, \text { as } x \leq \frac{n-2}{2} .
$$

Then $f(n, x)$ is monotonically decreasing on $x \leq \frac{n-2}{2}$. This completes the proof.

\section{Maximal (or minimal) irregularity of graphs in $\mathscr{T}_{n}^{\pi}$}

In this section, we explore some properties on $T_{\max }^{\pi}$ ( or $T_{\min }^{\pi}$ ), and find the corresponding trees $T_{\max }^{\pi}$ (or $\left.T_{m i n}^{\pi}\right)$.

\subsection{Properties on $T_{\max }^{\pi}$}

Lemma 3.1 Each path $v_{0} v_{1} \cdots v_{t} v_{t+1}$ with $d\left(v_{0}\right)=d\left(v_{t+1}\right)=1$ in $T_{\text {max }}^{\pi}$, has the following properties:

1. if $i$ is odd, then $d\left(v_{i}\right) \geq d\left(v_{t+1-i}\right) \geq d\left(v_{k}\right)$ for $i \leq k \leq t+1-i$;

2. if $i$ is even, then $d\left(v_{i}\right) \leq d\left(v_{t+1-i}\right) \leq d\left(v_{k}\right)$ for $i \leq k \leq t+1-i$. 
Proof: We prove the result by induction on $i$. For $i=1$, we will prove that $d\left(v_{1}\right) \geq d\left(v_{t}\right) \geq d\left(v_{k}\right)$ for $2 \leq k \leq t-1$. Suppose the opposite that $d_{T_{\max }^{\pi}}\left(v_{1}\right)<d_{T_{\max }^{\pi}}\left(v_{k}\right)$ for some $2 \leq k \leq t-1$. Let $T^{0}$ be a tree by applying $S\left(v_{1}, v_{k}\right)$ to $T_{\max }^{\pi}$. Clearly, $T^{0} \in \mathscr{T}_{n}^{\pi}$. Note that the edges $v_{0} v_{1}$ and $v_{k} v_{k+1}$ in $T_{\max }^{\pi}$ are transformed to the edges $v_{0} v_{k}$ and $v_{1} v_{k+1}$ in $T^{0}$, respectively. Hence we have

$$
\begin{aligned}
& \operatorname{irr}\left(T^{0}\right)-\operatorname{irr}\left(T_{\text {max }}^{\pi}\right)=\left|d_{T^{0}}\left(v_{0}\right)-d_{T^{0}}\left(v_{k}\right)\right|+\left|d_{T^{0}}\left(v_{1}\right)-d_{T^{0}}\left(v_{k+1}\right)\right| \\
& -\left|d_{T_{\max }^{\pi}}\left(v_{0}\right)-d_{T_{\max }^{\pi}}\left(v_{1}\right)\right|-\left|d_{T_{\max }^{\pi}}\left(v_{k}\right)-d_{T_{\max }^{\pi}}\left(v_{k+1}\right)\right| \\
& =\left|d_{T_{\max }^{\pi}}\left(v_{0}\right)-d_{T_{\max }^{\pi}}\left(v_{k}\right)\right|+\left|d_{T_{\text {max }}^{\pi}}\left(v_{1}\right)-d_{T_{\max }^{\pi}}\left(v_{k+1}\right)\right| \\
& -\left|d_{T_{\max }^{\pi}}\left(v_{0}\right)-d_{T_{\max }^{\pi}}\left(v_{1}\right)\right|-\left|d_{T_{\max }^{\pi}}\left(v_{k}\right)-d_{T_{\max }^{\pi}}\left(v_{k+1}\right)\right| \\
& =d_{T_{\max }^{\pi}}\left(v_{k}\right)-1+\left|d_{T_{\max }^{\pi}}\left(v_{1}\right)-d_{T_{\max }^{\pi}}\left(v_{k+1}\right)\right| \\
& -\left(d_{T_{\text {max }}^{\pi}}\left(v_{1}\right)-1\right)-\left|d_{T_{\text {max }}^{\pi}}\left(v_{k}\right)-d_{T_{\text {max }}^{\pi}}\left(v_{k+1}\right)\right| \text {, as } d_{T_{\text {max }}^{\pi}}\left(v_{0}\right)=1 \\
& >d_{T_{\max }^{\pi}}\left(v_{k}\right)-d_{T_{\max }^{\pi}}\left(v_{1}\right)-\left|d_{T_{\text {max }}^{\pi}}\left(v_{1}\right)-d_{T_{\max }^{\pi}}\left(v_{k}\right)\right| \\
& =d_{T_{\max }^{\pi}}\left(v_{k}\right)-d_{T_{\max }^{\pi}}\left(v_{1}\right)-\left(d_{T_{\max }^{\pi}}\left(v_{k}\right)-d_{T_{\max }^{\pi}}\left(v_{1}\right)\right) \\
& =0 \text {. }
\end{aligned}
$$

This is a contradiction. Hence $d_{T_{\max }^{\pi}}\left(v_{1}\right) \geq d_{T_{\max }^{\pi}}\left(v_{k}\right)$ for $2 \leq k \leq t-1$. At the same time, we have $d_{T_{\text {max }}^{\pi}}\left(v_{1}\right) \geq d_{T_{\text {max }}^{\pi}}\left(v_{t}\right)$. Similarly, we can prove that $d_{T_{\text {max }}^{\pi}}\left(v_{t}\right) \geq d_{T_{\text {max }}^{\pi}}\left(v_{k}\right)$ for $2 \leq k \leq t-1$. Hence, we conclude that $d_{T_{\max }^{\pi}}\left(v_{1}\right) \geq d_{T_{\max }^{\pi}}\left(v_{t}\right) \geq d_{T_{\max }^{\pi}}\left(v_{k}\right)$ for $1 \leq k \leq t$.

Now, assume that the result holds for other values. If $i \geq 2$ is even, assume that the result holds for any $l \leq i-1$. From that, if $l=i-1$ is odd, we have $d_{T_{\max }^{\pi}}\left(v_{i-1}\right) \geq d_{T_{\max }^{\pi}}\left(v_{t+2-i}\right) \geq d_{T_{\max }^{\pi}}\left(v_{k}\right)$ for $i \leq k \leq t+2-i$ and $i=1,2, \cdots,\lceil(t+1) / 2\rceil$. If $l=i$ is even, we should prove that $d_{T_{\max }^{m}}^{\max }\left(v_{i}\right) \leq$ $d_{T_{\text {max }}^{\pi}}\left(v_{t+1-i}\right) \leq d_{T_{\max }^{\pi}}\left(v_{k}\right)$ for $(i \leq k \leq t+1-i$ and $i=1,2, \cdots,\lceil(t+1) / 2\rceil)$. Suppose the opposite that $d_{T_{\text {max }}^{\pi}}\left(v_{i}\right)>d_{T_{\text {max }}^{\pi}}\left(v_{k}\right)$ for some $i+1 \leq k \leq t+1-i$. Let $T^{0}$ be a tree by applying $S\left(v_{i}, v_{k}\right)$ to $T_{\max }^{\pi}$. Clearly, $T^{0^{2}} \in \mathscr{T}_{n}^{\pi}$. Note that $v_{i-1} v_{i}$ and $v_{k} v_{k+1}$ in $T_{\max }^{\pi}$ are transformed to $v_{i-1} v_{k}$ and $v_{i} v_{k+1}$ in $T^{0}$, respectively. By the inductive hypothesis, we have $d_{T_{\max }^{\pi}}\left(v_{i-1}\right) \geq d_{T_{\text {max }}^{\pi}}\left(v_{k}\right)$ and $d_{T_{\text {max }}^{\pi}}\left(v_{i-1}\right) \geq d_{T_{\text {max }}^{\pi}}\left(v_{i}\right)$. Then we have

$$
\begin{aligned}
& \operatorname{irr}\left(T^{0}\right)-\operatorname{irr}\left(T_{\max }^{\pi}\right)=\left|d_{T^{0}}\left(v_{i-1}\right)-d_{T^{0}}\left(v_{k}\right)\right|+\left|d_{T^{0}}\left(v_{i}\right)-d_{T^{0}}\left(v_{k+1}\right)\right| \\
& -\left|d_{T_{\max }^{\pi}}\left(v_{i-1}\right)-d_{T_{\max }^{\pi}}\left(v_{i}\right)\right|-\left|d_{T_{\max }^{\pi}}\left(v_{k}\right)-d_{T_{\max }^{\pi}}\left(v_{k+1}\right)\right| \\
& =\left|d_{T_{\text {max }}^{\pi}}\left(v_{i-1}\right)-d_{T_{\text {max }}^{\pi}}\left(v_{k}\right)\right|+\left|d_{T_{\text {max }}^{\pi}}\left(v_{i}\right)-d_{T_{\text {max }}^{\pi}}\left(v_{k+1}\right)\right| \\
& -\left(d_{T_{\max }^{\pi}}\left(v_{i-1}\right)-d_{T_{\max }^{\pi}}\left(v_{i}\right)\right)-\left|d_{T_{\max }^{\pi}}\left(v_{k}\right)-d_{T_{\max }^{\pi}}\left(v_{k+1}\right)\right| \\
& =d_{T_{\text {max }}^{\pi}}\left(v_{i-1}\right)-d_{T_{\text {max }}^{\pi}}\left(v_{k}\right)+\left|d_{T_{\text {max }}^{\pi}}\left(v_{i}\right)-d_{T_{\text {max }}^{\pi}}\left(v_{k+1}\right)\right| \\
& -\left(d_{T_{\max }^{\pi}}\left(v_{i-1}\right)-d_{T_{\max }^{\pi}}\left(v_{i}\right)\right)-\left|d_{T_{\max }^{\pi}}\left(v_{k}\right)-d_{T_{\max }^{\pi}}\left(v_{k+1}\right)\right| \\
& >d_{T_{\text {max }}^{\pi}}\left(v_{i}\right)-d_{T_{\text {max }}^{\pi}}\left(v_{k}\right)-\left|d_{T_{\text {max }}^{\pi}}\left(v_{k}\right)-d_{T_{\text {max }}^{\pi}}\left(v_{i}\right)\right| \\
& =d_{T_{\max }^{\pi}}\left(v_{i}\right)-d_{T_{\max }^{\pi}}\left(v_{k}\right)-\left(d_{T_{\max }^{\pi}}\left(v_{i}\right)-d_{T_{\max }^{\pi}}\left(v_{k}\right)\right) \\
& =0 \text {, }
\end{aligned}
$$

a contradiction. Hence, $d_{T_{\text {max }}^{\pi}}\left(v_{i}\right) \leq d_{T_{\max }^{\pi}}\left(v_{k}\right)$ for any $i+1 \leq k \leq t+1-i$. At the same time, we have $d_{T_{\text {max }}^{\pi}}\left(v_{i}\right) \leq d_{T_{\max }^{\pi}}\left(v_{t+1-i}\right)$. Now we prove that $d_{T_{\text {max }}^{\pi}}\left(v_{t+1-i}\right) \leq d_{T_{\text {max }}^{\pi}}\left(v_{k}\right)$ for $i+1 \leq k \leq t+1-i$. Suppose the opposite that $d_{T_{\max }^{\pi}}\left(v_{t+1-i}\right)>d_{T_{\max }^{\pi}}\left(v_{k}\right)$ for $i+1 \leq k \leq t+1-i$. Let $T^{0}$ be the tree obtained by applying $S\left(v_{k}, v_{t+1-i}\right)$ to $T_{\max }^{\pi}$. Clearly, $T^{0} \in \mathscr{T}_{n}^{\pi}$. Note that $v_{k-1} v_{k}$ and $v_{t+1-i} v_{t+2-i}$ 
in $T_{\max }^{\pi}$ are transformed to $v_{k-1} v_{t+1-i}$ and $v_{k} v_{t+2-i}$ in $T^{0}$, respectively. Moreover, by the inductive hypothesis, we have $d_{T_{\max }^{\pi}}\left(v_{t+2-i}\right) \geq d_{T_{\max }^{\pi}}\left(v_{k}\right)$ and $d_{T_{\max }^{\pi}}\left(v_{t+2-i}\right) \geq d_{T_{\max }^{\pi}}\left(v_{t+1-i}\right)$. Then we have

$$
\begin{aligned}
& \operatorname{irr}\left(T^{0}\right)-\operatorname{irr}\left(T_{\max }^{\pi}\right)=\left|d_{T^{0}}\left(v_{k-1}\right)-d_{T^{0}}\left(v_{t+1-i}\right)\right|+\left|d_{T^{0}}\left(v_{k}\right)-d_{T^{0}}\left(v_{t+2-i}\right)\right| \\
& -\left|d_{T_{\max }^{\pi}}\left(v_{k-1}\right)-d_{T_{\text {max }}^{\pi}}\left(v_{k}\right)\right|-\left|d_{T_{\text {max }}^{\pi}}\left(v_{t+1-i}\right)-d_{T_{\text {max }}^{\pi}}\left(v_{t+2-i}\right)\right| \\
& =\left|d_{T_{\max }^{\pi}}\left(v_{k-1}\right)-d_{T_{\max }^{\pi}}\left(v_{t+1-i}\right)\right|+\left|d_{T_{\max }^{\pi}}\left(v_{k}\right)-d_{T_{\max }^{\pi}}\left(v_{t+2-i}\right)\right| \\
& -\left|d_{T_{\max }^{\pi}}\left(v_{k-1}\right)-d_{T_{\max }^{\pi}}\left(v_{k}\right)\right|-\left|d_{T_{\max }^{\pi}}\left(v_{t+1-i}\right)-d_{T_{\max }^{\pi}}\left(v_{t+2-i}\right)\right| \\
& =\left|d_{T_{\max }^{\pi}}\left(v_{k-1}\right)-d_{T_{\max }^{\pi}}\left(v_{t+1-i}\right)\right|+d_{T_{\max }^{\pi}}\left(v_{t+2-i}\right)-d_{T_{\max }^{\pi}}\left(v_{k}\right) \\
& -\left|d_{T_{\max }^{\pi}}\left(v_{k-1}\right)-d_{T_{\max }^{\pi}}\left(v_{k}\right)\right|-\left(d_{T_{\max }^{\pi}}\left(v_{t+2-i}\right)-d_{T_{\max }^{\pi}}\left(v_{t+1-i}\right)\right) \\
& >-\left|d_{T_{\max }^{\pi}}\left(v_{k}\right)-d_{T_{\max }^{\pi}}\left(v_{t+1-i}\right)\right|+d_{T_{\max }^{\pi}}\left(v_{t+1-i}\right)-d_{T_{\max }^{\pi}}\left(v_{k}\right) \\
& =\left(d_{T_{\max }^{\pi}}\left(v_{k}\right)-d_{T_{\max }^{\pi}}\left(v_{t+1-i}\right)\right)+d_{T_{\max }^{\pi}}\left(v_{t+1-i}\right)-d_{T_{\max }^{\pi}}\left(v_{k}\right) \\
& =0 \text {, }
\end{aligned}
$$

a contradiction. Hence we have $d_{T_{\max }^{\pi}}\left(v_{t+1-i}\right) \leq d_{T_{\max }^{\pi}}\left(v_{k}\right)$ for $i+1 \leq k \leq t+1-i$. Therefore, $d_{T_{\max }^{\pi}}\left(v_{i}\right) \leq d_{T_{\max }^{\pi}}\left(v_{t+1-i}\right) \leq d_{T_{\max }^{\pi}}\left(v_{k}\right)$ for $i \leq k \leq t+1-i$ and $i=1,2, \cdots,\lceil(t+1) / 2\rceil$.

The case for odd $i$ is similar. The proof is completed.

Let $v_{i, j}$ be the vertex whose closest leaf is at distance $i$, and let $v_{0, j}$ be a leaf in $T_{\max }^{\pi}$. For integers $i, j, k, l$, by Lemma 3.1 , we have the following:

Lemma 3.2 For $1 \leq i<j$, we have

1. $d_{T_{\max }^{\pi}}\left(v_{i, k}\right) \geq d_{T_{\max }^{\pi}}\left(v_{j, l}\right)$ for odd $i$;

2. $d_{T_{\max }^{\pi}}\left(v_{i, k}\right) \leq d_{T_{\max }^{\pi}}\left(v_{j, l}\right)$ for even $i$.

For a tree $T$, let $\mathscr{P}_{T}$ be the set of leaves in $T$, and $\mathscr{Q}_{T}$ be the set of vertices that adjacent to the leaves in $T$. Let $d^{\prime}=\min \left\{d(v), v \in \mathscr{Q}_{T}\right\}$ and $\mathscr{P}_{T}^{\prime}$ be the set of leaves whose adjacent vertices have degree $d^{\prime}$ in $T$.

Lemma 3.3 For trees $T$ and $T^{*}$ with root $r^{*}$, let $T^{\prime}$ and $T^{\prime \prime}$ be two trees obtained from $T$ by identifying the root $r^{*}$ of $T^{*}$ with $v^{\prime}$ and $v^{\prime \prime}$, respectively, where $v^{\prime} \in \mathscr{P}_{T}^{\prime}$ and $v^{\prime \prime} \in \mathscr{P}_{T} \backslash \mathscr{P}_{T}^{\prime}$. Then $\operatorname{irr}\left(T^{\prime}\right)>\operatorname{irr}\left(T^{\prime \prime}\right)$.

Proof: Let $v_{1}, v_{2} \in V(T)$ such that $v_{1} v^{\prime} \in E(T)$ and $v_{2} v^{\prime \prime} \in E(T)$. Note that $d_{T}\left(v_{1}\right)=d^{\prime}<d_{T}\left(v_{2}\right)$. Then we have

$$
\begin{aligned}
\operatorname{irr}\left(T^{\prime}\right)-\operatorname{irr}\left(T^{\prime \prime}\right)= & \left|d_{T^{\prime}}\left(v_{1}\right)-d_{T^{\prime}}\left(r^{*}\right)\right|+\left|d_{T^{\prime}}\left(v_{2}\right)-d_{T^{\prime}}\left(v^{\prime \prime}\right)\right| \\
& -\left|d_{T^{\prime \prime}}\left(v_{2}\right)-d_{T^{\prime \prime}}\left(r^{*}\right)\right|-\left|d_{T^{\prime \prime}}\left(v_{1}\right)-d_{T^{\prime \prime}}\left(v^{\prime}\right)\right| \\
= & \left|d_{T}\left(v_{1}\right)-\left(d_{T^{*}}\left(r^{*}\right)+1\right)\right|+\left|d_{T}\left(v_{2}\right)-d_{T}\left(v^{\prime \prime}\right)\right| \\
& -\left|d_{T}\left(v_{2}\right)-\left(d_{T^{*}}\left(r^{*}\right)+1\right)\right|-\left|d_{T}\left(v_{1}\right)-d_{T}\left(v^{\prime}\right)\right| \\
= & \left|d_{T}\left(v_{1}\right)-\left(d_{T^{*}}\left(r^{*}\right)+1\right)\right|+\left(d_{T}\left(v_{2}\right)-1\right) \\
& -\left|d_{T}\left(v_{2}\right)-\left(d_{T^{*}}\left(r^{*}\right)+1\right)\right|-\left(d_{T}\left(v_{1}\right)-1\right), \text { as } d_{T}\left(v^{\prime}\right)=d_{T}\left(v^{\prime \prime}\right)=1 \\
> & -\left|d_{T}\left(v_{2}\right)-d_{T}\left(v_{1}\right)\right|+d_{T}\left(v_{2}\right)-d_{T}\left(v_{1}\right) \\
= & -\left(d_{T}\left(v_{2}\right)-d_{T}\left(v_{1}\right)\right)+d_{T}\left(v_{2}\right)-d_{T}\left(v_{1}\right) \\
= & 0 .
\end{aligned}
$$


This completes the proof.

The following recursive algorithm can be used to construct the tree $T_{\max }^{\pi}$ with $\pi=\left\{d_{1}, d_{2}, \cdots, d_{m}\right\}$.

(1) If $m-1 \leq d_{m}$, then by Lemma 3.1 it is easy to get a tree $T_{\max }^{\pi}$ :

Rooted at $r$ with $d_{m}$ children with degrees $d_{1}, \cdots, d_{m-1}$ and $\underbrace{1, \cdots, 1}_{d_{m}-m+1}$;

(2) If $m-1 \geq d_{m}+1$, then by Lemma 3.2 , we see that the vertices in $\left\{v_{1, j} \mid j=1,2, \cdots\right\}$ take the largest degrees and they are adjacent to the vertices (in $\left\{v_{2, k} \mid k=1,2, \cdots\right\}$ ) with the smallest degrees. Construct the subtrees that contain vertices in $\left\{v_{0, i} \mid i=1,2, \cdots\right\},\left\{v_{1, j} \mid j=1,2, \cdots\right\}$ and $\left\{v_{2, k} \mid k=1,2, \cdots\right\}$ first. Note that by Lemma 3.1, we will let the larger degree vertex be adjacent to the smaller degree vertex whenever possible. Thus, we produce the following subtree $T_{1}$ :

Rooted at $r$ with $d_{m}-1$ children with degrees $d_{1}, \cdots, d_{d_{m}-1}$, where $r \in\left\{v_{2, k} \mid k=1,2, \cdots\right\}$ with degree $d_{m}$ in $T_{\text {max }}^{\pi}$, the children of $r$ are vertices in $\left\{v_{1, j} \mid j=1,2, \cdots\right\}$.

Note that removing $T_{1}$ (except the root) from $T_{\max }^{\pi}$ results in a new tree $S$ with degree sequence $\left\{d_{d_{m}}, \cdots, d_{m-1}\right\}$, in which Lemmas 3.1 and 3.2 still hold. Thus $S$ is a tree with the new degree sequence has the maximum irregularity.

(3) Now the only problem is how to attach $T_{1}$ to $S$ (by identifying the root of $T_{1}$ with a leaf of $S$ ). By Lemma 3.3. we should identify the root of $T_{1}$ with a vertex $v$ in $\mathscr{P}_{T}^{\prime}$ of $S$.

We now give an example which is a tree $T_{\max }^{\pi}$ with degree sequence $\pi=\{7,6,5,5,4,4,3,3,3,2\}$ to illustrate above mentioned process.

Firstly, by (2) we have the subtree $T_{1}$ and new degree sequence $\{6,5,5,4,4,3,3,3\}$. Then, we can find a tree with this new degree sequence has the maximum irregularity. Similarly, we also have the subtree $T_{2}$ and $T_{3}$ by (2). The remaining degree sequence $\{4,3\}$ satisfies (1), producing the tree $S$ with maximum irregularity, where $T_{1}, T_{2}, T_{3}$ and $S$ are shown in Fig. 3;

Secondly, attaching $T_{3}$ to $S$ (according to (3)) yields a tree with degree sequence $\{5,4,4,3,3\}$ has maximum irregularity. Now attaching $T_{2}$ to this new tree (according to (3)) yields a tree with degree sequence $\{6,5,5,4,4,3,3,3\}$ has the maximum irregularity. We then have a new $S$ (see Fig. 4);

Lastly, finding a leaf in the new $S$ (Fig. 4) whose neighbor has the smallest degree, attaching $T_{1}$ to the new $S$ as described above in (3) yields $T_{\max }^{\pi}$. However, $T_{\max }^{\pi}$ is not necessarily unique, two of them are shown in Fig. 5, both are achieved through our algorithm.

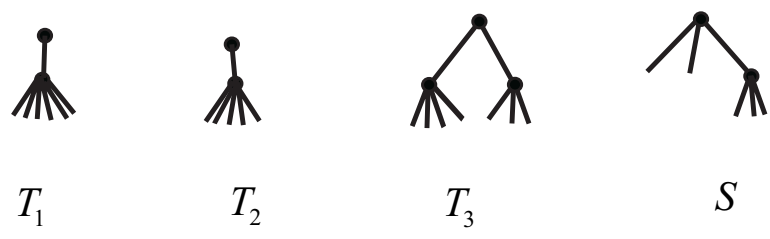

Fig. 3: Construction of subtrees. 


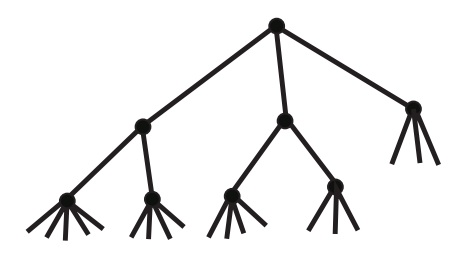

Fig. 4: Attaching subtrees to $S$.
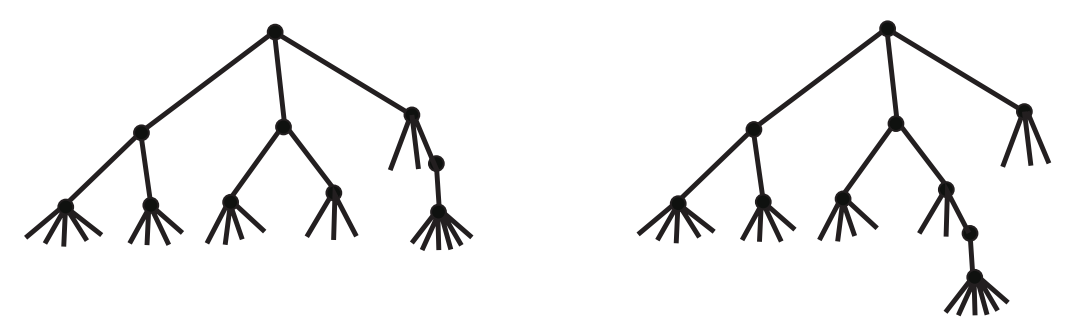

Fig. 5: Two trees $T_{\max }^{\pi}$ with the same degree sequence.

\subsection{Properties on $T_{\text {min }}^{\pi}$}

Lemma 3.4 Each path $v_{0} v_{1} v_{2} \cdots v_{t} v_{t+1}$ with $d\left(v_{0}\right)=d\left(v_{t+1}\right)=1$ in $T_{m i n}^{\pi}$, has the following properties:

$$
d\left(v_{i}\right) \leq d\left(v_{t+1-i}\right) \leq d\left(v_{k}\right),
$$

where $i+1 \leq k \leq t+1-i$ and $i=1,2, \cdots,\lceil(t+1) / 2\rceil$.

Proof: We prove the result by induction on $i$. For $i=1$, suppose the opposite that $d_{T_{m i n}^{\pi}}\left(v_{k}\right)<d_{T_{m i n}^{\pi}}\left(v_{1}\right)$ for $2 \leq k \leq t-1$. Let $T^{0}$ be the tree obtained by operating $S\left(v_{1}, v_{k}\right)$ to $T_{\min }^{\pi}$. Clearly, $T^{0} \in \mathscr{T}_{n}^{\pi}$. Note that the edges $v_{0} v_{1}$ and $v_{k} v_{k+1}$ in $T_{m i n}^{\pi}$ are transformed to the edges $v_{0} v_{k}$ and $v_{1} v_{k+1}$ in $T^{0}$, respectively. Then we have

$$
\begin{aligned}
\operatorname{irr}\left(T^{0}\right)-\operatorname{irr}\left(T_{m i n}^{\pi}\right)= & \left|d_{T^{0}}\left(v_{0}\right)-d_{T^{0}}\left(v_{k}\right)\right|+\left|d_{T^{0}}\left(v_{1}\right)-d_{T^{0}}\left(v_{k+1}\right)\right| \\
& -\left|d_{T_{m i n}^{\pi}}\left(v_{0}\right)-d_{T_{m i n}^{\pi}}\left(v_{1}\right)\right|-\left|d_{T_{m i n}^{\pi}}\left(v_{k}\right)-d_{T_{m i n}^{\pi}}\left(v_{k+1}\right)\right| \\
= & \left|d_{T_{m i n}^{\pi}}\left(v_{0}\right)-d_{T_{m i n}^{\pi}}\left(v_{k}\right)\right|+\left|d_{T_{m i n}^{\pi}}\left(v_{1}\right)-d_{T_{m i n}^{\pi}}\left(v_{k+1}\right)\right| \\
& -\left|d_{T_{m i n}^{\pi}}\left(v_{0}\right)-d_{T_{m i n}^{\pi}}\left(v_{1}\right)\right|-\left|d_{T_{m i n}^{\pi}}\left(v_{k}\right)-d_{T_{m i n}^{\pi}}\left(v_{k+1}\right)\right| \\
= & d_{T_{m i n}^{\pi}}\left(v_{k}\right)-1+\left|d_{T_{m i n}^{\pi}}\left(v_{1}\right)-d_{T_{m i n}^{\pi}}\left(v_{k+1}\right)\right| \\
& -\left(d_{T_{m i n}^{\pi}}\left(v_{1}\right)-1\right)-\left|d_{T_{m i n}^{\pi}}\left(v_{k}\right)-d_{T_{m i n}^{\pi}}\left(v_{k+1}\right)\right|, \text { as } d_{T_{m i n}^{\pi}}\left(v_{0}\right)=1 \\
< & d_{T_{m i n}^{\pi}}\left(v_{k}\right)-d_{T_{m i n}^{\pi}}\left(v_{1}\right)+\left|d_{T_{m i n}^{\pi}}\left(v_{1}\right)-d_{T_{m i n}^{\pi}}\left(v_{k}\right)\right| \\
= & d_{T_{m i n}^{\pi}}\left(v_{k}\right)-d_{T_{m i n}^{\pi}}\left(v_{1}\right)+d_{T_{m i n}^{\pi}}^{\pi}\left(v_{1}\right)-d_{T_{m i n}^{\pi}}\left(v_{k}\right) \\
= & 0 .
\end{aligned}
$$

This is a contradiction. Hence we have $d_{T_{m i n}^{\pi}}\left(v_{1}\right) \leq d_{T_{m i n}^{\pi}}\left(v_{k}\right)$ for $2 \leq k \leq t-1$. At the same time, we 
have $d_{T_{m i n}^{\pi}}\left(v_{1}\right) \leq d_{T_{m i n}^{\pi}}\left(v_{t}\right)$. Similarly, we also can verify $d_{T_{m i n}^{\pi}}\left(v_{t}\right) \leq d_{T_{m i n}^{\pi}}\left(v_{k}\right)$ for $2 \leq k \leq t-1$. Hence we have $d_{T_{m i n}^{\pi}}\left(v_{1}\right) \leq d_{T_{m i n}^{\pi}}\left(v_{t}\right) \leq d_{T_{m i n}^{\pi}}\left(v_{k}\right)$ for $2 \leq k \leq t$.

Now, assume that the result holds for any $l \leq i-1$. That is, we have $d_{T_{\min }^{\pi}}\left(v_{l}\right) \leq d_{T_{\min }^{\pi}}\left(v_{t+1-l}\right) \leq$ $d_{T_{\text {min }}^{\pi}}\left(v_{k}\right)$ for $l+1 \leq k \leq t+1-l, l=1,2, \cdots,\lceil(t+1) / 2\rceil$. For $l \stackrel{m}{=} i$, we have to prove that $d_{T_{m i n}^{\pi}}^{\text {min }}\left(v_{i}\right) \leq d_{T_{\text {min }}^{\pi}}\left(v_{t+1-i}\right) \leq d_{T_{\text {min }}^{\pi}}\left(v_{k}\right)$ for $i+1 \leq k \leq t+1-i$ and $i=1,2, \cdots,\lceil(t+1) / 2\rceil$. Suppose the opposite that $d_{T_{\text {min }}^{\pi}}\left(v_{i}\right)>d_{T_{\text {min }}^{\pi}}\left(v_{k}\right)$ for some $i+1 \leq k \leq t+1-i$. Let $T^{0}$ be the tree obtained by applying $S\left(v_{i}, v_{k}\right)$ to $T_{m i n}^{\pi}$. Clearly, $T^{0} \in \mathscr{T}_{n}^{\pi}$. Note that the edges $v_{i-1} v_{i}$ and $v_{k} v_{k+1}$ in $T_{m i n}^{\pi}$ are transformed to the edges $v_{i-1} v_{k}$ and $v_{i} v_{k+1}$ in $T^{0}$, respectively. Moreover, by the inductive hypothesis, we have $d_{T_{m i n}^{\pi}}\left(v_{i-1}\right) \leq d_{T_{m i n}^{\pi}}\left(v_{k}\right)$ and $d_{T_{m i n}^{\pi}}\left(v_{i-1}\right) \leq d_{T_{m i n}^{\pi}}\left(v_{i}\right)$. Then we have

$$
\begin{aligned}
\operatorname{irr}\left(T^{0}\right)-\operatorname{irr}\left(T_{m i n}^{\pi}\right)= & \left|d_{T^{0}}\left(v_{i-1}\right)-d_{T^{0}}\left(v_{k}\right)\right|+\left|d_{T^{0}}\left(v_{i}\right)-d_{T^{0}}\left(v_{k+1}\right)\right| \\
& -\left|d_{T_{m i n}^{\pi}}\left(v_{i-1}\right)-d_{T_{m i n}^{\pi}}\left(v_{i}\right)\right|-\left|d_{T_{m i n}^{\pi}}\left(v_{k}\right)-d_{T_{m i n}^{\pi}}\left(v_{k+1}\right)\right| \\
= & \left|d_{T_{m i n}^{\pi}}\left(v_{i-1}\right)-d_{T_{m i n}^{\pi}}\left(v_{k}\right)\right|+\left|d_{T_{m i n}^{\pi}}\left(v_{i}\right)-d_{T_{m i n}^{\pi}}\left(v_{k+1}\right)\right| \\
& -\left(d_{T_{m i n}^{\pi}}\left(v_{i}\right)-d_{T_{m i n}^{\pi}}\left(v_{i-1}\right)\right)-\left|d_{T_{m i n}^{\pi}}\left(v_{k}\right)-d_{T_{m i n}^{\pi}}\left(v_{k+1}\right)\right| \\
= & d_{T_{m i n}^{\pi}}\left(v_{k}\right)-d_{T_{m i n}^{\pi}}\left(v_{i-1}\right)+\left|d_{T_{m i n}^{\pi}}\left(v_{i}\right)-d_{T_{m i n}^{\pi}}\left(v_{k+1}\right)\right| \\
& -\left(d_{T_{m i n}^{\pi}}\left(v_{i}\right)-d_{T_{m i n}^{\pi}}\left(v_{i-1}\right)\right)-\left|d_{T_{m i n}^{\pi}}\left(v_{k}\right)-d_{T_{m i n}^{\pi}}\left(v_{k+1}\right)\right| \\
< & d_{T_{m i n}^{\pi}}\left(v_{k}\right)-d_{T_{m i n}^{\pi}}\left(v_{i}\right)+\left|d_{T_{m i n}^{\pi}}\left(v_{i}\right)-d_{T_{m i n}^{\pi}}\left(v_{k}\right)\right| \\
= & d_{T_{m i n}^{\pi}}\left(v_{k}\right)-d_{T_{m i n}^{\pi}}\left(v_{i}\right)+d_{T_{m i n}^{\pi}}\left(v_{i}\right)-d_{T_{m i n}^{\pi}}\left(v_{k}\right) \\
= & 0 .
\end{aligned}
$$

This is a contradiction. Hence we have $d_{T_{m i n}^{\pi}}\left(v_{i}\right) \leq d_{T_{m i n}^{\pi}}\left(v_{k}\right)$ for $i+1 \leq k \leq t+1-i$. At the same time, we have $d_{T_{m i n}^{\pi}}\left(v_{i}\right) \leq d_{T_{m i n}^{\pi}}\left(v_{t+1-i}\right)$. By the same argument as above, we have $d_{T_{m i n}^{\pi}}\left(v_{t+1-i}\right) \leq$ $d_{T_{m i n}^{\pi}}\left(v_{k}\right)$ for $i+1 \leq k \leq t+1-i$. Hence we have $d_{T_{m i n}^{\pi}}\left(v_{i}\right) \leq d_{T_{m i n}^{\pi}}\left(v_{t+1-i}\right) \leq d_{T_{m i n}^{\pi}}\left(v_{k}\right)$ for $i+1 \leq k \leq t+1-i, i=1,2, \cdots,\lceil(t+1) / 2\rceil$. This completes the proof.

Lemma 3.5 For any $T \in \mathscr{T}_{n}^{\pi}$ with $u v, x y \in E(T)$ and $u y, x v \notin E(T)$, let $T^{0}=T-u v-x y+u y+x v$. If $d_{T}(u) \geq d_{T}(x) \geq d_{T}(y) \geq d_{T}(v)$, then $\operatorname{irr}\left(T^{0}\right)=\operatorname{irr}(T)$.

Proof: Note that $d(u) \geq d(x) \geq d(y) \geq d(v)$. Then we have

$$
\begin{aligned}
\operatorname{irr}\left(T^{0}\right)-\operatorname{irr}(T)= & \left|d_{T^{0}}(u)-d_{T^{0}}(y)\right|+\left|d_{T^{0}}(x)-d_{T^{0}}(v)\right| \\
& -\left|d_{T}(u)-d_{T}(v)\right|-\left|d_{T}(x)-d_{T}(y)\right| \\
= & \left(d_{T}(u)-d_{T}(y)\right)+\left(d_{T}(x)-d_{T}(v)\right) \\
& -\left(d_{T}(u)-d_{T}(v)\right)-\left(d_{T}(x)-d_{T}(y)\right) \\
= & 0 .
\end{aligned}
$$

This completes the proof.

Suppose that the degrees of the non-leaf vertices are given. The greedy tree is achieved by the following "greedy algorithm" [12, 13]:

(i) label the vertex with the largest degree as $v_{0,1}$ (the root); 
(ii) label the neighbors of $v_{0,1}$ as $v_{1,1}, v_{1,2}, \cdots$, assign the largest degrees available to them such that $d\left(v_{1,1}\right) \geq d\left(v_{1,2}\right) \geq \cdots$

(iii) label the neighbors of $v_{1,1}$ (except $v_{0,1}$ ) as $v_{2,1}, v_{2,2}, \cdots$, such that they take all the largest degrees available and that $d\left(v_{2,1}\right) \geq d\left(v_{2,2}\right) \geq \cdots$, then do the same for $v_{1,2}, v_{1,3}, \cdots$;

(iv) repeat (iii) for all the newly labelled vertices, always start with the neighbors of the labelled vertex with largest degree whose neighbors are not labelled yet.

Theorem 3.1 Among trees in $\mathscr{T}_{n}^{\pi}$, there exists a greedy tree with minimum irregularity.

Proof: Firstly, by the definition of the greedy tree with a given degree sequence, we easily to see that the greedy tree satisfies the conditions in Lemma 3.4 However, there are many trees for which these conditions hold. Then by Lemma 3.5 , the greedy trees with minimum irregularity are constructed among these trees. This completes the proof.

Remark 3.1 In fact, there are many trees different from the greedy tree with a given degree sequence minimize $\operatorname{irr}(T)$. Following is an example which are two $T_{\min }^{\pi} \mathrm{s}$. One is obtained by the greedy algorithm with degree sequence $\pi=\{4,4,3,3,3,3,3,2,2,2,2\}$, also it is a greedy tree. The other one is not a greedy tree with the same degree sequence, which are shown in Fig. 6
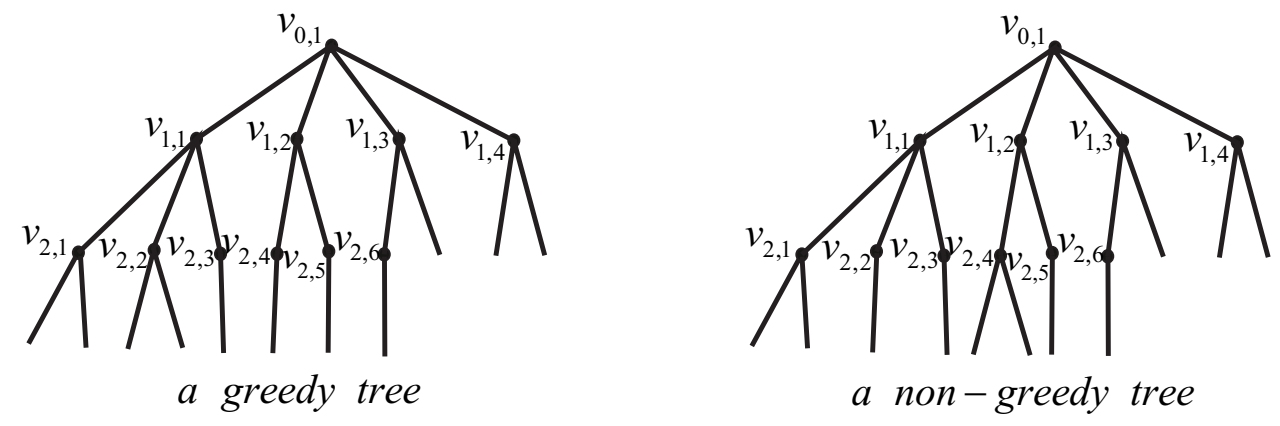

Fig. 6: A greedy tree and a non-greedy tree with degree sequence $\pi=\{4,4,3,3,3,3,3,2,2,2,2\}$.

\section{Maximal (or minimal) irregularity of graphs in $\mathscr{B}_{n}^{k}$}

In this section, we explore some properties on $B_{\max }^{k}$ (or $B_{\min }^{k}$ ), and construct the corresponding trees $B_{\max }^{k}\left(\right.$ or $\left.B_{\min }^{k}\right)$.

We now explore some properties for trees in $\mathscr{B}_{n}^{k}$. For $T \in \mathscr{B}_{n}^{k}$, let $n_{i}$ be the number of vertices of degree $i$ in $T$ for $i=1,2, \cdots, \Delta_{1}$. Then we have

$$
\sum_{i=1}^{\Delta_{1}} n_{i}=n \quad \text { and } \quad \sum_{i=1}^{\Delta_{1}} i n_{i}=2(n-1) .
$$


Note that

$$
2(n-1)=\sum_{i=1}^{\Delta_{1}} i n_{i}=n_{1}+2 n_{2}+\sum_{i=3}^{\Delta_{1}} i n_{i} \geq n_{1}+n_{2}+3 k=n-k+3 k=n+2 k .
$$

This implies that

Proposition 4.1 For any tree $T \in \mathscr{B}_{n}^{k}, k(T) \leq \frac{n-2}{2}$.

Moreover, note that

$$
\begin{aligned}
2(n-1)=\sum_{i=1}^{\Delta_{1}} i n_{i} & =n_{1}+2 n_{2}+\sum_{i=3}^{\Delta_{1}} i n_{i} \\
& \geq n_{1}+n_{2}+3\left(k-n_{\Delta_{1}}\right)+\Delta_{1} n_{\Delta_{1}} \\
& =n-k+3\left(k-n_{\Delta_{1}}\right)+\Delta_{1} n_{\Delta_{1}} \\
& =n+2 k+\left(\Delta_{1}-3\right) n_{\Delta_{1}} \geq n+2 k+\left(\Delta_{1}-3\right) .
\end{aligned}
$$

This leads to the following conclusion.

Proposition 4.2 For any tree $T \in \mathscr{B}_{n}^{k}, \Delta_{1}(T) \leq n-2 k+1$.

\subsection{Properties on $B_{\max }^{k}$}

Lemma 4.1 For $1<k \leq \frac{n-2}{2}$, $B_{\max }^{k}$ has the following properties:

1. $B_{\max }^{k}$ contains no vertex with degree 2 ;

2. $B_{\max }^{k}$ contains at most one vertex of degree larger than 3 .

Proof: (1) Assume that there exists $v \in V\left(B_{\max }^{k}\right)$ such that $d_{B_{\max }^{k}}(v)=2$. Let $N_{B_{\max }^{k}}(v)=\left\{w_{1}, w_{2}\right\}$. Then $d_{B_{\max }^{k}}\left(w_{1}\right) \geq 2$ or $d_{B_{\max }^{k}}\left(w_{2}\right) \geq 2$. Let $u \in V\left(B_{\max }^{k}\right)$ with $d_{B_{\max }^{k}}(u) \stackrel{\max }{=} \Delta_{1}\left(B_{\max }^{k}\right)$. Let $N_{B_{\max }^{k}}(u)=\left\{u_{1}, u_{2}, \cdots, u_{\Delta_{1}}\right\}$. If $u \in N_{B_{m a x}^{k}}(v)$, let $T^{\prime}$ be the tree obtained from $B_{\max }^{k}$ by Separating an edge $u v$. Clearly, $T^{\prime} \in \mathscr{B}_{n}^{k}$. Then Lemma 2.1 implies that $\operatorname{irr}\left(T^{\prime}\right)>\operatorname{irr}\left(B_{\text {max }}^{k}\right)$, a contradiction. If $u \notin N_{B_{\max }^{k}}(v)$, then there exists a path contains $u$ and $v$ in $B_{\text {max }}^{k}$. Let $v$ be the vertex connected to $u$ via $w_{1}$ with $d_{B_{\max }^{k}}\left(w_{1}\right) \geq 2$. Let $T^{\prime}=B_{\max }^{k}-v w_{2}+u w_{2}$. Clearly, $T^{\prime} \in \mathscr{B}_{n}^{k}$. Hence we have

$$
\begin{aligned}
& \operatorname{irr}\left(T^{\prime}\right)-\operatorname{irr}\left(B_{\max }^{k}\right) \\
& =\left|d_{T^{\prime}}(u)-d_{T^{\prime}}\left(w_{2}\right)\right|+d_{B_{\max }^{k}}(u)+\left|d_{T^{\prime}}(v)-d_{T^{\prime}}\left(w_{1}\right)\right| \\
& -\left|d_{B_{\text {max }}^{k}}(v)-d_{B_{\text {max }}^{k}}\left(w_{1}\right)\right|-\left|d_{B_{\text {max }}^{k}}(v)-d_{B_{\text {max }}^{k}}\left(w_{2}\right)\right| \\
& =\left|d_{B_{\max }^{k}}^{k}(u)+1-d_{B_{\max }^{k}}\left(w_{2}\right)\right|+d_{B_{\max }^{k}}(u) \\
& +\left|d_{B_{\max }^{k}}(v)-1-d_{B_{\max }^{k}}\left(w_{1}\right)\right|-\left|d_{B_{\max }^{k}}(v)-d_{B_{\max }^{k}}\left(w_{1}\right)\right| \\
& -\left|d_{B_{\max }^{k}}(v)-d_{B_{\text {max }}^{k}}\left(w_{2}\right)\right| \\
& =d_{B_{\max }^{k}}(u)+1-d_{B_{\max }^{k}}\left(w_{2}\right)+d_{B_{\max }^{k}}(u) \\
& +\left|2-1-d_{B_{\max }^{k}}\left(w_{1}\right)\right|-\left(d_{B_{\max }^{k}}\left(w_{1}\right)-2\right)-\left|d_{B_{\max }^{k}}\left(w_{2}\right)-2\right| \text {, as } d_{B_{\max }^{k}}(v)=2 \\
& =2 d_{B_{\text {max }}^{k}}(u)+2-d_{B_{\text {max }}^{k}}\left(w_{2}\right)-\left|d_{B_{\text {max }}^{k}}\left(w_{2}\right)-2\right| \\
& >0 \text {, }
\end{aligned}
$$


a contradiction. Hence the result follows.

(2) Assume that there exists two vertices $u, v \in V\left(B_{\max }^{k}\right)$ such that $d_{B_{\max }^{k}}(u)=\Delta_{1}\left(B_{\max }^{k}\right)>3$ and $d_{B_{\max }^{k}}(v)>3$. Let $d_{B_{\max }^{k}}(v)=s$ and $N_{B_{\max }^{k}}(v)=\left\{v_{1}, v_{2}, \cdots, v_{s}\right\}$. If $u \in N_{B_{\max }^{k}}(v)$, without loss of generality, we assume that $u=v_{s}$. Let $T^{\prime}=B_{\max }^{k}-\left\{v v_{1}, v v_{2}, \cdots, v v_{s-3}\right\}+\left\{u v_{1}, u v_{2}, \cdots, u v_{s-3}\right\}$. Clearly, $T^{\prime} \in \mathscr{B}_{n}^{k}$. Hence we have

$$
\begin{aligned}
& \operatorname{irr}\left(T^{\prime}\right)-\operatorname{irr}\left(B_{\max }^{k}\right) \\
= & \sum_{i=1}^{s-3}\left[\left|d_{T^{\prime}}(u)-d_{T^{\prime}}\left(v_{i}\right)\right|-\left|d_{B_{\max }^{k}}(v)-d_{B_{\max }^{k}}\left(v_{i}\right)\right|\right] \\
& +2(s-3)+(s-3)\left(d_{B_{\max }^{k}}(u)-1\right) \\
& +\sum_{i=s-2}^{s-1}\left[\left|d_{T^{\prime}}(v)-d_{T^{\prime}}\left(v_{i}\right)\right|-\left|d_{B_{\max }^{k}}(v)-d_{B_{\max }^{k}}\left(v_{i}\right)\right|\right] \\
= & \sum_{i=1}^{s-3}\left[\left|d_{B_{\max }^{k}}(u)+s-3-d_{B_{\max }^{k}}\left(v_{i}\right)\right|-\left|d_{B_{\max }^{k}}(v)-d_{B_{\max }^{k}}\left(v_{i}\right)\right|\right] \\
& +2(s-3)+(s-3)\left(d_{B_{\max }^{k}}(u)-1\right) \\
& +\sum_{i=s-2}^{s-1}\left[\left|d_{B_{\max }^{k}}(v)-(s-3)-d_{B_{\max }^{k}}\left(v_{i}\right)\right|-\left|d_{B_{\max }^{k}}(v)-d_{B_{\max }^{k}}\left(v_{i}\right)\right|\right] \\
>\quad & -(s-3)\left|d_{B_{\max }^{k}}(v)-d_{B_{\max }^{k}}(u)-(s-3)\right| \\
& +(s-3)\left(d_{B_{\max }^{k}}(u)+1\right)-2(s-3) \\
= & -(s-3)\left(d_{B_{\max }^{k}}(u)-3\right)+(s-3)\left(d_{B_{\max }^{k}}(u)-1\right), \text { as } d_{B_{\max }^{k}}(v)=s \\
= & 2(s-3) \\
> & 0,
\end{aligned}
$$

a contradiction. If $u \notin N_{B_{\max }^{k}}(v)$, let $T^{\prime}=B_{\max }^{k}-\left\{v v_{1}, v v_{2}, \cdots, v v_{s-3}\right\}+\left\{u v_{1}, u v_{2}, \cdots, u v_{s-3}\right\}$. Clearly, $T^{\prime} \in \mathscr{B}_{n}^{k}$. By the same argument as above, we have $\operatorname{irr}\left(T^{\prime}\right)>\operatorname{irr}\left(B_{\max }^{k}\right)$. This is also a contradiction. Hence the result follows.

Combing Proposition 4.1 and Lemma 4.1, we then have the following.

Theorem 4.1 For $1 \leq k \leq \frac{n-2}{2}, B_{\max }^{k}$ has the degree sequence $\pi=\{n-2 k+1, \underbrace{3, \cdots, 3}_{k-1}\}$.

Lemma 4.2 For any tree $T \in \mathscr{T}_{n}^{\pi}$ with degree sequence $\pi=\{\Delta_{1}, \underbrace{\Delta_{2}, \cdots, \Delta_{2}}_{k-1}\}$, where $k \leq \frac{n-2}{2}$, then $\operatorname{irr}(T)=\Delta_{1}^{2}+\Delta_{1}\left(1-2 \Delta_{2}\right)+\left[2 n-2+(1-k) \Delta_{2}\right]\left(\Delta_{2}-1\right)$.

Proof: Note that $T$ with degree sequence $\pi=\{\Delta_{1}, \underbrace{\Delta_{2}, \cdots, \Delta_{2}}_{k-1}\}$. Then $T$ has $t=2(n-1)-\Delta_{1}-$ $(k-1) \Delta_{2}$ leaves. Assume that $u_{1} \in V(T)$ with $d\left(u_{1}\right)=\Delta_{1}$. Note that for any tree $T \in \mathscr{T}_{n}^{\pi}$, there are 
$\Delta_{1}$ disjoint pendent paths which begin with the vertex $u_{1}$. If one of them is $P_{1}=u_{1} u_{2} \cdots u_{i} v_{j}$, where $d\left(u_{i}\right)=\Delta_{2}$ for $2 \leq i \leq k$, and $d\left(v_{j}\right)=1$ for $1 \leq j \leq t$. Then we have

$$
\begin{aligned}
\operatorname{irr}\left(P_{1}\right) & =\sum_{x y \in E\left(P_{1}\right)}|d(x)-d(y)| \\
& =\left|d\left(u_{1}\right)-d\left(u_{2}\right)\right|+\left|d\left(u_{2}\right)-d\left(u_{3}\right)\right|+\cdots+\left|d\left(u_{i-1}\right)-d\left(u_{i}\right)\right|+\left|d\left(u_{i}\right)-d\left(v_{j}\right)\right| \\
& =d\left(u_{1}\right)-d\left(u_{2}\right)+d\left(u_{2}\right)-d\left(u_{3}\right)+\cdots+d\left(u_{i-1}\right)-d\left(u_{i}\right)+d\left(u_{i}\right)-d\left(v_{j}\right) \\
& =d\left(u_{1}\right)-d\left(v_{j}\right) \\
& =\Delta_{1}-1 ;
\end{aligned}
$$

if one of them is $P_{2}=u_{1} v_{j}$, where $d\left(v_{j}\right)=1$. Then we have $\operatorname{irr}\left(P_{2}\right)=\left|d\left(u_{1}\right)-d\left(v_{j}\right)\right|=\Delta_{1}-1$. Obviously, there is $\Delta_{1}\left(\Delta_{1}-1\right)$ contributes to $\operatorname{irr}(T)$, by the $\Delta_{1}$ disjoint pendent paths which begin with the vertex $u_{1}$. By the construction of trees, there are leaving $t-\Delta_{1}$ pendent vertices which just adjacent to the vertices with degree $\Delta_{2}$, then they have $\left(t-\Delta_{1}\right)\left(\Delta_{2}-1\right)$ contributes to $\operatorname{irr}(T)$. No matter how to construct the tree $T$, there are some edges as the edge $u_{i} u_{j}$ with $d\left(u_{i}\right)=d\left(u_{j}\right)=\Delta_{2}$, and their balance is 0 as $\left|d\left(u_{i}\right)-d\left(u_{j}\right)\right|=0$. Therefore, we have

$$
\begin{aligned}
\operatorname{irr}(T) & =\Delta_{1}\left(\Delta_{1}-1\right)+\left(t-\Delta_{1}\right)\left(\Delta_{2}-1\right)+0 \\
& =\Delta_{1}^{2}-\Delta_{1} \Delta_{2}+t\left(\Delta_{2}-1\right) \\
& =\Delta_{1}^{2}-\Delta_{1} \Delta_{2}+\left[2(n-1)-\Delta_{1}-(k-1) \Delta_{2}\right]\left(\Delta_{2}-1\right) \\
& =\Delta_{1}^{2}+\Delta_{1}\left(1-2 \Delta_{2}\right)+\left[2 n-2+(1-k) \Delta_{2}\right]\left(\Delta_{2}-1\right) .
\end{aligned}
$$

This completes the proof.

Remark 4.1 In fact, Lemma 4.2 also holds for $\Delta_{1}=\Delta_{2}$. That is, for any tree $T \in \mathscr{T}_{n}^{\pi}$ with degree sequence $\{\underbrace{\Delta_{1}, \cdots, \Delta_{1}}_{k}\}$, where $k \leq \frac{n-2}{2}$, then $\operatorname{irr}(T)=-k \Delta_{1}^{2}+(2 n+k-2) \Delta_{1}-2 n+2$.

By Theorem 4.1 and Lemma 4.2 , we then have the following.

Theorem 4.2 For any tree $T \in \mathscr{B}_{n}^{k}$, where $1 \leq k \leq \frac{n-2}{2}$, we have

$$
\operatorname{irr}(T) \leq n^{2}+(1-4 k) n+4 k^{2}-2,
$$

and the equality holds if and only if $T$ has degree sequence $\pi=\{n-2 k+1, \underbrace{3, \cdots, 3}_{k-1}\}$.

Moreover, by Theorem 4.2 and Lemma 2.2, we have the following.

Corollary 4.1 $\operatorname{irr}\left(B_{\max }^{1}\right)>\operatorname{irr}\left(B_{\max }^{2}\right)>\cdots>\operatorname{irr}\left(B_{\text {max }}^{\left\lfloor\frac{n-2}{2}\right\rfloor}\right)$.

\subsection{Properties on $B_{\text {min }}^{k}$}

Lemma 4.3 For $1 \leq k \leq \frac{n-2}{2}, \Delta_{1}\left(B_{\min }^{k}\right)<4$. 
Proof: Suppose that there exists $u \in V\left(B_{\min }^{k}\right)$ such that $d_{B_{m i n}^{k}}(u)=\Delta_{1}\left(B_{\text {min }}^{k}\right) \geq 4$. Let $N(u)=$ $\left\{u_{1}, u_{2}, \cdots, u_{\Delta_{1}-2}, v_{i-1}, v_{i+1}\right\}$. Let $P=v_{0} v_{1}, \cdots, v_{i-1} u\left(\stackrel{m=}{=} v_{i}\right) v_{i+1}, \cdots, v_{l}$ be the longest path in $B_{m i n}^{k}$ that contains $u$. Let $w_{1}$ be a pendent vertex connected to $u$ via $u_{1}$ (it is possible that $w_{1} \equiv u_{1}$ ) (Fig.77). Let $T^{1}=B_{m i n}^{k}-u u_{2}+u_{2} w_{1}$. Clearly, $T^{1} \in \mathscr{B}_{n}^{k}$. Then we have

$$
\begin{aligned}
\operatorname{irr}\left(T^{1}\right)-\operatorname{irr}\left(B_{m i n}^{k}\right)= & \left|d_{T^{\prime}}\left(w_{1}\right)-d_{T^{\prime}}\left(u_{2}\right)\right|-1-\left(d_{B_{m i n}^{k}}(u)-1\right) \\
& -\left|d_{B_{m i n}^{k}}(u)-d_{B_{m i n}^{k}}^{k}\left(u_{2}\right)\right| \\
= & \left|d_{B_{m i n}^{k}}\left(w_{1}\right)+1-d_{B_{m i n}^{k}}^{k}\left(u_{2}\right)\right|-d_{B_{m i n}^{k}}(u) \\
& -\left(d_{B_{m i n}^{k}}^{k}(u)-d_{B_{m i n}^{k}}\left(u_{2}\right)\right) \\
= & \left|2-d_{B_{m i n}^{k}}^{k}\left(u_{2}\right)\right|-2 d_{B_{m i n}^{k}}^{k}(u)+d_{B_{m i n}^{k}}\left(u_{2}\right), \text { as } d_{B_{m i n}^{k}}\left(w_{1}\right)=1 \\
< & 0,
\end{aligned}
$$

a contradiction. This completes the proof.
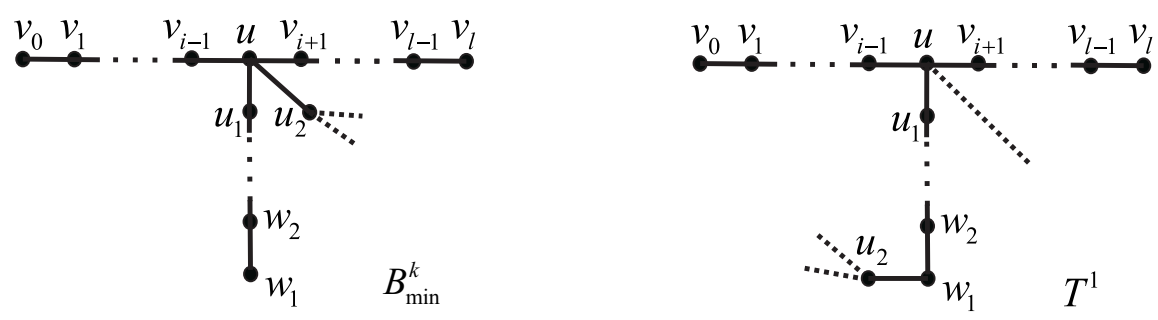

Fig. 7: Trees $B_{m i n}^{k}$ and $T^{1}$ in the proof of Lemma 4.3

Theorem 4.3 For $1 \leq k \leq \frac{n-2}{2}, B_{\min }^{k}$ has the degree sequence $\pi=\{\underbrace{3, \cdots, 3}_{k}, \underbrace{2, \cdots, 2}_{n-2 k-2}\}$.

Proof: By Lemma 4.3 we assume that $B_{\min }^{k}$ has degree sequence $\{\underbrace{3, \cdots, 3}_{k}, \underbrace{2, \cdots, 2}_{x}, \underbrace{1, \cdots, 1}_{y}\}$, where $x, y$ are integers. Note that $k+x+y=n$ and $3 k+2 x+y=2(n-1)$. Those yield that $x=n-2 k-2$ and $y=k+2$. Hence the result holds.

Theorem 4.4 For any $T \in \mathscr{B}_{n}^{k}$, where $k \leq \frac{n-2}{2}$, then we have

$$
\operatorname{irr}(T) \geq 2 k+4
$$

and the equality holds if and only if $T \cong T_{\min }^{\pi}$ with $\pi=\{\underbrace{3, \cdots, 3}_{k}, \underbrace{2, \cdots, 2}_{n-2 k-2}\}$.

Proof: By Theorem 4.3 for any $T \in \mathscr{B}_{n}^{k}$, we have $\operatorname{irr}(T) \geq \operatorname{irr}\left(B_{\min }^{k}\right)=\operatorname{irr}\left(T_{\text {min }}^{\pi}\right)$ with $\pi=$ $\{\underbrace{3, \cdots, 3}_{k}, \underbrace{2, \cdots, 2}_{n-2 k-2}\}$. Moreover, by Theorem 3.1 for $\pi=\{\underbrace{3, \cdots, 3}_{k}, \underbrace{2, \cdots, 2}_{n-2 k-2}\}$, there exists a greedy tree 
$T^{*}$ such that $\operatorname{irr}\left(T^{*}\right)=\operatorname{irr}\left(T_{m i n}^{\pi}\right)$. By the construct of the greedy tree $T^{*}$ with $\pi=\{\underbrace{3, \cdots, 3}_{k}, \underbrace{2, \cdots, 2}_{n-2 k-2}\}$, we have

$$
\begin{aligned}
\operatorname{irr}\left(T^{*}\right) & =\sum_{u v \in E\left(T^{*}\right)}|d(u)-d(v)| \\
& =3(3-1)+(k-1)(3-2)(3-1)+0 \\
& =2 k+4 .
\end{aligned}
$$

Hence the result follows.

From the proof of Theorem 4.4, we know that $\operatorname{irr}\left(T^{*}\right)$ is monotonically increasing on $k$. Hence we have the following.

Corollary 4.2 $6=\operatorname{irr}\left(B_{\min }^{1}\right)<\operatorname{irr}\left(B_{\min }^{2}\right)<\cdots<\operatorname{irr}\left(B_{\min }^{\left\lfloor\frac{n-2}{2}\right\rfloor}\right)$.

\section{Acknowledgements}

The authors would like to thank the anonymous referees for their constructive corrections and valuable comments on this paper, which have considerably improved the presentation of this paper.

\section{References}

[1] H. Abdo, N. Cohen, D. Dimitrov, Bounds and computation of irregularity of a graph, Filomat, 28(2014), 13151322.

[2] H. Abdo, D. Dimitrov, The irregularity of graphs under graph operations, Discuss. Math. Graph Theory, 34(2014), 263-278.

[3] M. Albertson, The irregularity of a graph, Ars Combin., 46(1997), 219-225.

[4] C. Berge, Graphs and Hypergraphs, 2nd edn., North-Holland, Amsterdam, 1976.

[5] G. Chartrand, P. Erdös, How to define an irregularity graph, Coll. Math. J., 19(1988), 36-42.

[6] P. Hansen, H. Mélot, Variable neighborhood search for extremal graphs 9. Bounding the irregularity of a graph, DIMACS Ser. Discrete Math. Theoret. Comput. Sci., 69(2005), 253-264.

[7] M.A. Henning, D. Rautenbach, On the irregularity of bipartite graphs, Discrete Math., 307(2007), 1467-1472.

[8] Y. Liu, J.-B. Lv, The effects on the irregularity of graphs with some transformations, J. Minnan Normal University, 4(2014), 8-14.

[9] W. Luo, B. Zhou, On the irregularity of trees and unicyclic graphs with given matching number, Util. Math., 83(2010), 141-147.

[10] M. Tavakoli, F. Rahbarnia, M. Mirzavaziri, A.R. Ashrafi, I. Gutman, Extremely irregular graphs, Kragujevac J. Math., 37(2013), 135-139.

[11] M. Tavakoli, F. Rahbarnia, A. R. Ashrafi, Some new results on irregularity of graphs, J. Appl. Math. Inform., 32(2014), 675-685.

[12] H. Wang, Extremal trees with given degree sequence for the Randić index, Discrete Math., 308(2008), $3407-$ 3411.

[13] R. Xing, B. Zhou, Extremal trees with fixed degree sequence for atom-bond connectivity index, Filomat, 26(2012), 683-688.

[14] B. Zhou, On irregularity of graphs, Ars Combin., 88(2008), 55-64. 